\title{
Quantitative experiments on supersaturated solutions for the undergraduate thermodynamics laboratory
}

\author{
J Güémez ${ }^{1}, C$ Fiolhais ${ }^{2}$ and M Fiolhais ${ }^{2}$ \\ ${ }^{1}$ Departamento de Física Aplicada, Universidad de Cantabria, E-39005 Santander, Spain \\ 2 Departamento de Física and Centro de Física Computacional, Universidade de Coimbra, \\ P-3004-516 Coimbra, Portugal \\ E-mail: guemezj@unican.es, tcarlos@teor.fis.uc.pt and tmanuel@teor.fis.uc.pt
}

Received 9 July 2004, in final form 10 September 2004

Published 26 October 2004

Online at stacks.iop.org/EJP/26/25

\begin{abstract}
Quantitative experiments on supersaturated solutions are presented. They serve to introduce topics such as the solubility curve and irreversible phase transitions. Experimental results are analysed with the aid of Hess's law. These kind of experiments, which in the present case employed sodium acetate trihydrate, may be chosen as a student's project in experimental thermodynamics.
\end{abstract}

\section{Introduction}

Examples of metastable states are the so-called supercooled liquids, which occur when the liquid phase (a local minimum of the Gibbs function) does exist, though the solid phase (absolute minimum of the Gibbs function) is more stable under the same conditions [1]. In [1], we have illustrated experimentally some phenomena undergone by supercooled liquids. Closely related are phenomena occurring in supersaturated solutions [2]. The solubility of a solid in a liquid is described by a solubility curve, which gives, for an equilibrium state, the maximal amount of solid, $m_{\mathrm{D}}(t)$, that can be dissolved in a certain amount of liquid, as a function of the temperature. For a saturated solution, the mass of the solute is given by the solubility curve, while for an unsaturated solution there is less solute and for a supersaturated solution there is more. A supersaturated solution is metastable: it may become a mixture of solid in equilibrium with a saturated solution.

Along the same lines of our previous paper on supercooled liquids [1], we present, in this work, a series of experiments on supersaturated solutions. In our experiments we used supersaturated solutions of sodium acetate trihydrate $\left(\mathrm{NaCH}_{3} \mathrm{COO} \cdot 3 \mathrm{H}_{2} \mathrm{O}\right)$, hereafter 

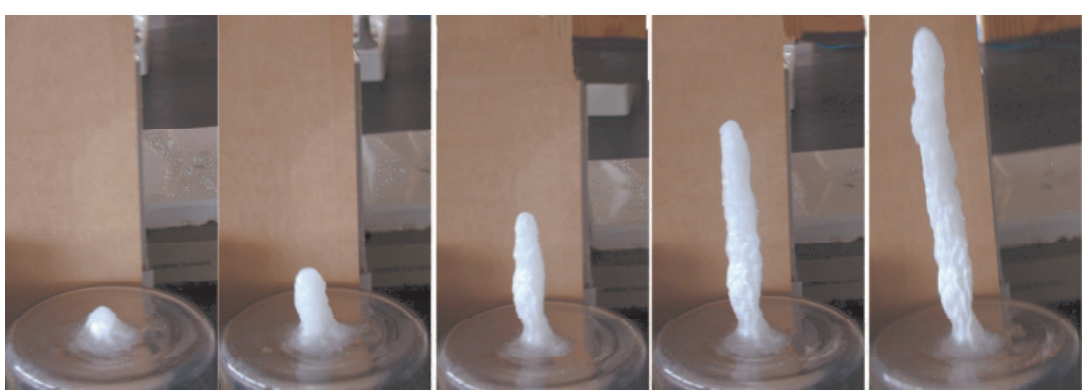

Figure 1. Stalagmite of solid SAT when a supersaturated SAT solution is poured over a seed crystal. As the stalagmite grows, the temperature increases.

(This figure is in colour only in the electronic version)

referred to as SAT, in water, since they are easily prepared. A supersaturated aqueous solution of SAT may remain indefinitely, but, when it is slightly perturbed (e.g., by the presence of a SAT crystal — seed — or by the gentle perturbation caused, for instance, by the probe of a digital thermometer when it is slightly shaken), crystallization occurs. One observes that the homogeneous liquid suddenly separates into a solid and a saturated SAT-water solution while the system temperature increases. There is a parallelism between these phenomena and solidification in supercooled liquids [1].

Though supersaturated solutions provide experiments which are visually appealing and even amazing (see figure 1), the description of quantitative experiments in this topic is scarce in the literature, and this fact was indeed one of the motivations for the present work.

In principle, the theory describing the processes is not involved, but, as in other experimental works, the results may contain an element of surprise.

When a mass $m$ of SAT is dissolved into a fixed amount of hot water of mass $m_{\mathrm{W}}$ and then cooled down, a supersaturated solution at room temperature, $t_{\mathrm{i}}$, is obtained. When part of the dissolved salt crystallizes, a certain amount of heat is released (crystallization enthalpy), increasing the temperature of both the solid and of the saturated SAT-water solution. A final temperature, $t_{\mathrm{f}}$, is eventually reached.

In order to know the mass of salt which remains dissolved after crystallization, one has to insert $t_{\mathrm{f}}$ in the equation of the solubility curve, i.e., one has to calculate $m_{\mathrm{D}}\left(t_{\mathrm{f}}\right)$. The mass of the crystallized salt is therefore:

$$
m_{\mathrm{S}}\left(t_{\mathrm{f}}\right)=m-m_{\mathrm{D}}\left(t_{\mathrm{f}}\right) .
$$

The enthalpy balance equation may be written as

$$
\Delta h_{\text {cry }}(\bar{t}) m_{\mathrm{S}}(\bar{t})+m_{\mathrm{S}}\left(t_{\mathrm{f}}\right) c_{\mathrm{S}}(\bar{t})\left(t_{\mathrm{f}}-t_{\mathrm{i}}\right)+\left[m_{\mathrm{W}}+m_{\mathrm{D}}\left(t_{\mathrm{f}}\right)\right] c_{\mathrm{SS}}\left(t_{\mathrm{f}}-t_{i}\right)=0
$$

where $\Delta h_{\text {cry }}(\bar{t})$ is the enthalpy of crystallization for SAT in the interval of temperatures $(\bar{t}$ is an intermediate temperature between $t_{\mathrm{i}}$ and $\left.t_{\mathrm{f}}\right), c_{\mathrm{S}}(\bar{t})$ is the (average) specific heat of SAT and $c_{\mathrm{SS}}$ is the specific heat for saturated SAT-water solution, which is assumed to be constant.

The released heat is the enthalpy of crystallization. The term enthalpy of solidification is not completely appropriate here, since our experiments are done by crystallizing the salt out of the solution and not by freezing the liquid. In section 3, we shall further discuss this point.

The laboratory work should be divided into three steps: (i) previous measurement of some quantities when they are not available in the literature, (ii) preparation of supersaturated solutions, well in advance, and the carrying out of crystallization experiments and (iii) analysis of experimental results. 
Table 1. For different thermostat temperatures, $t$, listed are the SAT masses, $m_{\mathrm{D}}$, which saturate $20 \mathrm{~cm}^{3}$ of water.

\begin{tabular}{ll}
\hline$t /{ }^{\circ} \mathrm{C}$ & $m_{\mathrm{D}} / \mathrm{g}$ \\
\hline $15.6 \pm 0.1$ & $22.85 \pm 0.02$ \\
$18.5 \pm 0.1$ & $24.31 \pm 0.02$ \\
$21.5 \pm 0.1$ & $25.36 \pm 0.02$ \\
$25.8 \pm 0.1$ & $26.55 \pm 0.02$ \\
$31.9 \pm 0.1$ & $29.08 \pm 0.02$ \\
$36.1 \pm 0.1$ & $32.30 \pm 0.02$ \\
$39.3 \pm 0.1$ & $36.10 \pm 0.02$ \\
$44.6 \pm 0.1$ & $45.86 \pm 0.02$ \\
\hline
\end{tabular}

This paper is organized as follows. In section 2 the SAT-water solubility curve is obtained, as well as the SAT specific heat and saturated SAT-water solution specific heat. Additionally, some data needed in the analysis are reported. In section 3, experimental temperatures for supersaturated SAT-water solution crystallization are presented and SAT specific heat and enthalpy of crystallization are obtained. These results are discussed using the SAT-water solubility curve and Hess's law. In section 4, we summarize the method and present the conclusions.

\section{Solubility curve and specific heats}

\subsection{Solubility curve}

To obtain the SAT-water solubility curve we introduced $20 \mathrm{~cm}^{3}$ of distilled water in a test tube, which was placed inside a thermostat, and measured the mass of dissolved $\mathrm{SAT}^{3}$. When a small crystal remains undissolved at the bottom of the test tube, the mass which remains from a previously known amount is measured in order to obtain the dissolved mass. Table 1 shows our experimental results.

With the data of table 1, we obtained the following polynomial fit for the SAT solubility curve (in $20 \mathrm{~cm}^{3}$ of water)

$$
m_{\mathrm{D}}(t)=-2.24+3.00 t-0.114 t^{2}+0.00159 t^{3} .
$$

This expression is valid around and above room temperature.

\subsection{Specific heats}

When a SAT supersaturated solution precipitates, solid SAT and saturated SAT solution remain in the test tube. In the final analysis, the solid SAT and the SAT saturated solution specific heats (both at constant pressure) will be important. We performed a set of calorimetric experiments in order to obtain those specific heats.

The present case is similar to that discussed in [1] with the sum of all enthalpy variations vanishing (see equation (1) of that reference). Therefore, the water equivalent of the calorimeter for these kind of experiments, $m_{\mathrm{C}}$, is obtained as in [1], yielding

$$
m_{\mathrm{C}}=\frac{m_{\mathrm{HW}}\left(t_{\mathrm{HW}}^{\mathrm{i}}-t_{\mathrm{HW}}^{\mathrm{f}}\right)}{\left(t^{\mathrm{f}}-t^{\mathrm{i}}\right)}-m_{\mathrm{p}} .
$$

\footnotetext{
3 When the salt is added, the temperature inside the test tube decreases initially; but, when the solution is close to saturation, the temperature does not change anymore. This observation can be used to recognize the vicinity of saturation.
} 
Table 2. Results of calorimetric experiments. A mass of hot water, $m_{\mathrm{HW}}$, at initial temperature $t_{\mathrm{HW}}^{\mathrm{i}}$ is placed inside a calorimeter. In a metallic recipient with a thin wall a mass $m_{\mathrm{p}}$ of cold water $\{1\}$, SAT supersaturated solution $\{2\}$, sodium chloride $(\mathrm{Na} \mathrm{Cl})\{3\}$ and sodium acetate trihydrate $\{4\}$, at initial temperature $t^{\mathrm{i}}$, are placed in thermal contact with the hot water. After 3 min of gentle stirring, the final temperatures of hot water, $t_{\mathrm{HW}}^{\mathrm{f}}$, and of the substance in the metallic recipient, $t^{\mathrm{f}}$, are measured.

\begin{tabular}{lllllll}
\hline & $m_{\mathrm{HW}} / \mathrm{g}$ & $t_{\mathrm{HW}}^{\mathrm{i}} /{ }^{\circ} \mathrm{C}$ & $m_{\mathrm{p}} / \mathrm{g}$ & $t^{\mathrm{i}} /{ }^{\circ} \mathrm{C}$ & $t_{\mathrm{HW}}^{\mathrm{f}} /{ }^{\circ} \mathrm{C}$ & $t^{\mathrm{f}} /{ }^{\circ} \mathrm{C}$ \\
\hline$\{1\}$ & 135.7 & 45.0 & 141.3 & 20.0 & 32.2 & 31.6 \\
$\{2\}$ & 180.7 & 45.0 & 129.8 & 20.1 & 31.7 & 31.0 \\
$\{3\}$ & 183.1 & 45.0 & 196.6 & 21.5 & 39.3 & 37.6 \\
$\{4\}$ & 134.6 & 44.0 & 140.1 & 21.6 & 37.9 & 34.0 \\
\hline
\end{tabular}

All quantities in this expression are introduced in the caption of table 2.

Also from $\Delta H=0$, and in a similar way, the specific heat, $c$, can be obtained:

$$
c=c_{\mathrm{W}}\left[\frac{m_{\mathrm{HW}}\left(t_{\mathrm{HW}}^{\mathrm{i}}-t_{\mathrm{HW}}^{\mathrm{f}}\right)}{m_{\mathrm{p}}\left(t^{\mathrm{f}}-t^{\mathrm{i}}\right)}-\frac{m_{\mathrm{C}}}{m_{\mathrm{p}}}\right],
$$

where $c_{\mathrm{W}}=4.18 \mathrm{~J} \mathrm{~g}^{-1} \mathrm{~K}^{-1}$ is the water specific heat. Our calorimetric data are given in table 2 .

Using equation (4) with data $\{1\}$ from table 2 we obtained $m_{\mathrm{C}}=8.4 \pm 0.6 \mathrm{~g}$. Using equation (5) with data $\{2\}$ in table 2 , we obtained $c_{\mathrm{SS}}=3.47 \mathrm{~J} \mathrm{~g}^{-1}{ }^{\circ} \mathrm{C}^{-1}$ for the heat capacity of SAT saturated solution. After performing three experiments of this kind, we obtained $c_{\mathrm{SS}}=3.45 \pm 0.15 \mathrm{~J} \mathrm{~g}^{-1}{ }^{\circ} \mathrm{C}^{-1}$.

Since sodium acetate trihydrate is solid (powder), we checked this simple calorimetric method with $\mathrm{NaCl}$ (tiny crystals). According to the data in the literature, the heat capacity of $\mathrm{NaCl}$ is $c_{P}(\mathrm{NaCl})=0.902 \mathrm{~J} \mathrm{~g}^{-1}{ }^{\circ} \mathrm{C}^{-1}$, at $298 \mathrm{~K}$. Using equation (5) with data $\{3\}$ in table 2, we obtained $c_{P}(\mathrm{NaCl})=0.87 \pm 0.05 \mathrm{~J} \mathrm{~g}^{-1}{ }^{\circ} \mathrm{C}^{-1}$ (the error bars result from a set of three experiments). Using equation (5) with data $\{4\}$ in table 2 , we obtained $c_{\mathrm{S}}=1.72 \mathrm{~J} \mathrm{~g}^{-1}{ }^{\circ} \mathrm{C}^{-1}$ for the sodium acetate trihydrate heat capacity. After performing three different experiments of this kind, we arrived at $c_{\mathrm{S}}=1.75 \pm 0.08 \mathrm{~J} \mathrm{~g}^{-1}{ }^{\circ} \mathrm{C}^{-1}$.

\subsection{Data in the literature}

According to the literature [3], the heat capacity of solid SAT (whose molecular weight is $136.08 \mathrm{~g} \mathrm{~mol}^{-1}$ ) at constant pressure is $c_{P}=229.0 \mathrm{~J} \mathrm{~mol}^{-1} \mathrm{~K}^{-1}=1.68 \mathrm{~J} \mathrm{~g}^{-1} \mathrm{~K}^{-1}$ at $298.15 \mathrm{~K}$ and $c_{P}=322.0 \mathrm{~J} \mathrm{~mol}^{-1} \mathrm{~K}^{-1}=2.37 \mathrm{~J} \mathrm{~g}^{-1} \mathrm{~K}^{-1}$ at $325 \mathrm{~K}$.

The SAT enthalpy of fusion is $\Delta h_{\text {fus }}=20.250 \mathrm{~kJ} \mathrm{~mol}^{-1}=148.10 \mathrm{~J} \mathrm{~g}^{-1}$, and the entropy of fusion $\Delta s_{\text {fus }}=61.0 \mathrm{~J} \mathrm{~mol}^{-1} \mathrm{~K}^{-1}=0.448 \mathrm{~J} \mathrm{~g}^{-1} \mathrm{~K}^{-1}$ at the normal fusion temperature $T_{\text {fus }}=331.7 \mathrm{~K}$.

\section{The experiments}

To obtain supersaturated SAT solutions, we poured different amounts of SAT into different test tubes, all containing $20 \mathrm{~cm}^{3}$ of distilled water. All solutions were gently heated, immersing the test tube into hot water. Solutions were filtered to avoid impurities and then slowly cooled down to room temperature.

Afterwards, a metallic probe of a digital thermometer was merged into each solution. A small disturbance induces the salt solidification and the observed temperature increase was 


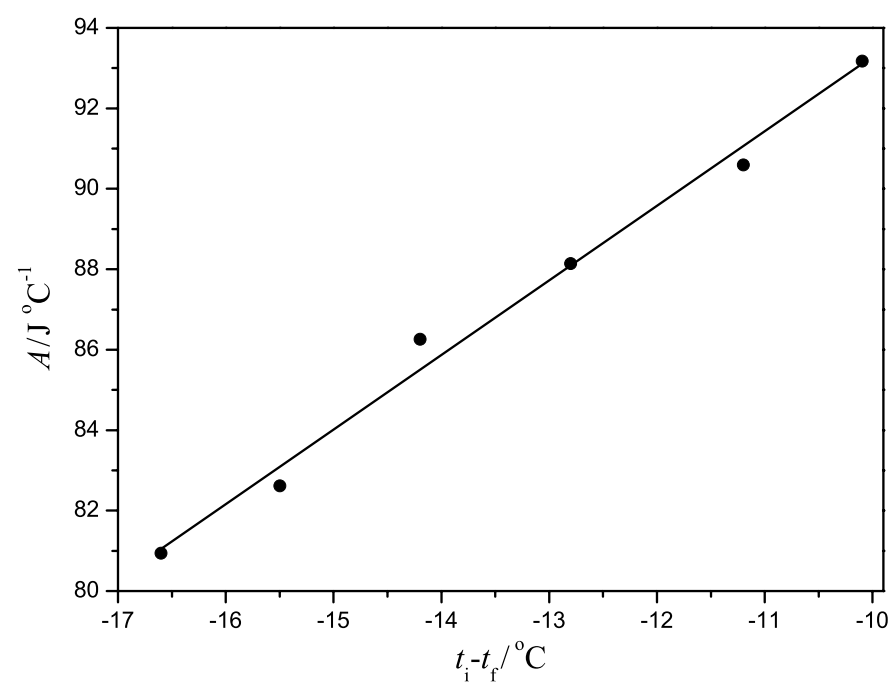

Figure 2. Graphic of $A$, given by equation (7) versus $t_{\mathrm{i}}-t_{\mathrm{f}}$. The values $m_{\mathrm{D}}\left(t_{\mathrm{f}}\right)$ can be obtained using equation (3) and $t_{\mathrm{f}}$ given in table 3 . The least-squares fit gives $A=1.85\left(t_{\mathrm{i}}-t_{\mathrm{f}}\right)+111.8$.

Table 3. After dissolving the mass $m$ of SAT into $20 \mathrm{~g}$ of water and gently heating, solutions were filtered and slowly cooled down to room temperature $t_{\mathrm{i}}$. A disturbance induces salt to crystallize and the final temperature, $t_{\mathrm{f}}$, was measured.

\begin{tabular}{lll}
\hline$m / \mathrm{g}$ & $t_{\mathrm{i}} /{ }^{\circ} \mathrm{C}$ & $t_{\mathrm{f}} /{ }^{\circ} \mathrm{C}$ \\
\hline $46.50 \pm 0.02$ & $20.6 \pm 0.1$ & $30.7 \pm 0.1$ \\
$49.81 \pm 0.02$ & $20.5 \pm 0.1$ & $31.7 \pm 0.1$ \\
$54.89 \pm 0.02$ & $20.4 \pm 0.1$ & $33.2 \pm 0.1$ \\
$60.01 \pm 0.02$ & $20.5 \pm 0.1$ & $34.7 \pm 0.1$ \\
$65.02 \pm 0.02$ & $19.9 \pm 0.1$ & $35.4 \pm 0.1$ \\
$70.51 \pm 0.02$ & $20.2 \pm 0.1$ & $36.8 \pm 0.1$ \\
\hline
\end{tabular}

measured ( 3 min were necessary for the temperature to stabilize). Our data for several test tubes are given in table 3 .

For $m_{\mathrm{W}}=20 \mathrm{~g}$, in order to know $m_{\mathrm{D}}\left(t_{\mathrm{f}}\right)$ after crystallization, we insert each final experimental temperature $t_{\mathrm{f}}$, given in table 3 , in the solubility equation, equation (3). Then, using equation (1), $m_{\mathrm{S}}\left(t_{\mathrm{f}}\right)$ was obtained.

Equation (2) may still be written as

$$
A=c_{\mathrm{S}}\left(t_{\mathrm{i}}-t_{\mathrm{f}}\right)-\Delta h_{\text {cry }}
$$

where the quantities

$$
A=\frac{c_{\mathrm{SS}}\left(t_{\mathrm{f}}-t_{\mathrm{i}}\right)}{m_{\mathrm{S}}\left(t_{\mathrm{f}}\right)}\left[m_{\mathrm{W}}+m_{\mathrm{D}}\left(t_{\mathrm{f}}\right)\right] \quad \text { and } \quad \Delta t=t_{\mathrm{f}}-t_{\mathrm{i}}
$$

can be determined from the data in table 3 and other above-mentioned data.

Figure 2 represents $A$ versus $\left(t_{\mathrm{i}}-t_{\mathrm{f}}\right)$. From this we get $c_{\mathrm{S}}=1.85 \mathrm{~J} \mathrm{~g}^{-1}{ }^{\circ} \mathrm{C}^{-1}$ and $\Delta h_{\text {cry }}=-111.8 \mathrm{~J} \mathrm{~g}^{-1}$.

According to the literature, $c_{\mathrm{S}}$ varies with temperature as $0.026 \mathrm{~J} \mathrm{~g}^{-1} \mathrm{~K}^{-2}, c_{\mathrm{S}}(T)=$ $1.68+0.026(T-298) \mathrm{J} \mathrm{g}^{-1} \mathrm{~K}^{-1}$, and at $32{ }^{\circ} \mathrm{C}$ it can be estimated to be $c_{\mathrm{S}}=1.86 \mathrm{~J} \mathrm{~g}^{-1} \mathrm{~K}^{-1}$.

On the other hand, $\Delta h_{\text {cry }}$ is quite different from the previously reported data on $\Delta h_{\text {fus }}$. Actually, SAT-water supersaturated solution crystallization is slightly different from 
the liqxuid-solid SAT phase transition. Given the aqueous solution character of liquid SAT, it is possible to guess that the enthalpy of crystallization will not be very different from the enthalpy of fusion, $\Delta h_{\text {cry }}\left(T_{\text {fus }}\right) \approx-\Delta h_{\text {fus }}\left(T_{\text {fus }}\right)$.

The experimental result for the enthalpy of crystallization at temperature $T$ may be obtained using Hess's law. It is given by

$$
\Delta h_{\text {cry }}\left(T_{\text {fus }}\right)=\int_{T_{\text {fus }}}^{T} c_{\mathrm{SS}} \mathrm{d} T+\Delta h_{\text {cry }}(T)+\int_{T}^{T_{\text {fus }}} c_{\mathrm{S}} \mathrm{d} T,
$$

and, assuming $c_{\mathrm{SS}}$ to be constant and $c_{\mathrm{S}}(T)$ as given above,

$\Delta h_{\text {cry }}(T)=\Delta h_{\text {cry }}\left(T_{\text {fus }}\right)+\left(c_{\text {SS }}-1.68\right)\left(T_{\text {fus }}-T\right)-\frac{0.026}{2}\left[\left(T_{\text {fus }}-298\right)^{2}-(T-298)^{2}\right]$.

By a similar calculation, the entropy of solidification at temperature $T$ is given by

$$
\Delta s_{\text {cry }}(T)=\Delta s_{\text {cry }}\left(T_{\text {fus }}\right)+\left(c_{\mathrm{SS}}-1.68\right) \ln \left(\frac{T_{\text {fus }}}{T}\right)-0.026\left(T_{\text {fus }}-T\right)+7.75 \ln \left(\frac{T_{\text {fus }}}{T}\right) .
$$

Using the previously reported data and assuming that the temperature for sodium acetate supersaturated solution solidification is around $32^{\circ} \mathrm{C}(T \approx 305 \mathrm{~K})$, we get $\Delta h_{\text {cry }}(305)=-112$ $\mathrm{J} \mathrm{g}^{-1}$ and $\Delta s_{\text {cry }}(305)=-0.333 \mathrm{~J} \mathrm{~g}^{-1} \mathrm{~K}^{-1}$, with $\Delta s_{\text {cry }}(305)=-0.333>\Delta h_{\text {cry }}(305) /$ $305=-0.367$, according to Clausius' inequality and showing that this kind of crystallization is an irreversible process.

\section{Conclusions}

Our first goal was to determine the final temperature of a supersaturated solution that evolves to a final state with a solid phase in equilibrium with a saturated liquid. The analysis requires knowing the specific heats of the solid, which depends on the temperature of the saturated solution, which is assumed constant, and the crystallization enthalpy. Due to the large difference between the specific heats of the solid and of the saturated solution, according to Hess's law, the crystallization enthalpy strongly depends on the final temperature. In order to predict the final temperature, one has to perform a self-consistent calculation for which a good guess of the final temperature is required.

In this work we studied phenomena occurring in supersaturated solutions, using sodium acetate trihydrate as working substance. In order to obtain the final temperature it was necessary to know the solubility curve, so that this function had to be previously obtained in a set of experiments. Moreover, the specific heats of the solid and the saturated solution were obtained also in a set of calorimetric experiments. The use of the Hess law and Clausius inequality showed that the phase transition was indeed irreversible.

The material required to perform these kind of experiments is easily available, making them suitable for student's projects in laboratory thermodynamics.

\section{Acknowledgments}

This work was supported in part by the Ministry of Science and Technology of Spain, grant number HP02-8 and by the 'Spanish-Portuguese Integrated Actions' programme.

\section{References}

[1] Güémez J, Fiolhais C and Fiolhais M 2002 Reproducing Black's experiments: freezing point depression and supercooling of water Eur. J. Phys. 23 83-91 
[2] Ahmad J 2000 Crystallization from a supersaturated solution of sodium acetate J. Chem. Educ. 771446 and references therein

[3] Meisingset K K and Gronvøld F 1984 Thermodynamic properties and phase transitions of salt hydrates between 270 and $400 \mathrm{~K} . \mathrm{CH}_{3} \mathrm{CO}_{2} \mathrm{Na} \cdot 3 \mathrm{H}_{2} \mathrm{O}, \mathrm{CH}_{3} \mathrm{CO}_{2} \mathrm{Li} \cdot 2 \mathrm{H}_{2} \mathrm{O}$, and $\left(\mathrm{CH}_{3} \mathrm{CO}_{2}\right)_{2} \mathrm{Mg} \cdot 4 \mathrm{H}_{2} \mathrm{O}$ J. Chem. Thermodyn. $16523-36$

Sturley K R 1932 Fresh data on the latent heats and heat conductivities of some aquacrystalline compounds J. Chem. Ind. Trans. Commun. 51 271T-3T NIST Chemistry Web Book http://webbook.nist.gov/chemistry/ 